Revue internationale P.M.E.

Économie et gestion de la petite et moyenne entreprise

Revue

internationale

PME

\title{
Le temps de la périphérie
}

\section{Pasquale Coppola}

Volume 2, numéro 2-3, 1989

URI : https://id.erudit.org/iderudit/1007931ar

DOI : https://doi.org/10.7202/1007931ar

Aller au sommaire du numéro

Éditeur(s)

Presses de l'Université du Québec

ISSN

0776-5436 (imprimé)

1918-9699 (numérique)

Découvrir la revue

Citer cet article

Coppola, P. (1989). Le temps de la périphérie. Revue internationale P.M.E., 2(2-3), 133-144. https://doi.org/10.7202/1007931ar

\section{Résumé de l'article}

L'article analyse le récent succès des PME de l'Italie du Centre et du Nord-Est. On signale le développement d'un ensemble de sous-systèmes industriels locaux, peu reliés entre eux mais pourvus de solides liens d'interdépendance interne. Parmi les raisons fondementales à la base des performances surprenantes, on indique certaines caractéristiques particulières du cadre social de ces régions ainsi que du rôle d'un tissu urbain organisé en réseau. d'utilisation que vous pouvez consulter en ligne.

https://apropos.erudit.org/fr/usagers/politique-dutilisation/ 


\title{
Le temps de la périphérie:
}

\author{
Pasquale COPPOLA* \\ Institut Universitaire Oriental de Naples
}

\begin{abstract}
RÉSUMÉ
L'article analyse le récent succès des PME de l'Italie du Centre et du Nord-Est. On signale le développement d'un ensemble de sous-systèmes industriels locaux, peu reliés entre eux mais pourvus de solides liens d'interdépendance interne. Parmi les raisons fondementales à la base des performances surprenantes, on indique certaines caractéristiques particulières du cadre social de ces régions ainsi que du rôle d'un tissu urbain organisé en réseau.
\end{abstract}

\begin{abstract}
The paper presents an analysis of the recent success of small and medium-sized businesses in central and north-eastern Italy. It indicates the development of a set of local industrial subsystems having few links between them but possessing solid internal interdependence ties. Among the basic reasons underlying the surprising performances, particular characteristics of the social framework of the regions and the role played by a networked urban web are highlighted.
\end{abstract}

\section{RESUMEN}

El articulo analiza el reciente éxito de las PyMl en Italia del Centro y del Noreste. Se senala el desarrollo de un conjunto de subsistemas industriales locales, poco relacionados entre ellos pero provistos de solidos vinculos de interdependencia interna. Entre las razones fundamentales de la base de los rendimientos sorprendentes, se indicas ciertas caracteristicas particulares del àmbito social de esas regiones, al igual que el papel de un trama urbano organizado en red.

* Cet article est tiré, en partie, d'une communication présentée au XXIV· congrès de géographie italienne, les 25-31 mars 1985 à Turin et en partie d'un article publié dans le «Bollettino della Società geografica italiana», 4,6,1989.

* Pasquale Coppola est professeur de Géographie politique et économique à la Faculté de Sciences Politiques de l'Institut Universitaire Oriental de Naples. Il étudie les problèmes du développement régional, surtout dans les pays méditerranéens, et les rapports entre structures de la production et le processus d'urbanisation. Adresse : Dipartimento di Scienze Sociali. Istituto Universitario Orientale - Pizza San Giovanni Maggiore Pignatelli, $30-80100$ Napoli. 


\section{Chronique d'une communication future}

En 1957, les Italiens ont commencé à construire pierre par pierre ou brique par brique leur premier boom économique. Mais leurs efforts ont donné d'étranges résultats. Cette même année, un professeur américain d'économie, John-Kenneth Galbraith (1958), décrivait la fébrilité presque mythique de cette production accélérée comme les pulsations du coeur de «l'affluent society» (des Etats-Unis). Une telle thèse fit beaucoup de bruit; elle fut regardée avec soupçon et on l'attribua au snobisme génial des gens de Harvard et à la «rigueur carrée» de le vieille Amérique.

La théorie de Galbraith de l'hégémonie du centre dans la production mondiale et de la fin du contrôle politique de l'économie ne pouvait pas, toutefois, permettre d'anticiper la transition qui s'annonçait. Mais les signes de cette transition étaient déjà dans l'air : les derniers vertiges, mais aussi le début du refus de la consommation de masse, la pression immodérée sur les ressources naturelles qui suscitait le cri d'alarme du Club de Rome, la possibilité accrue du prochain conflit mondial préparé dans les laboratoires, et de nouvelles méthodes de travail «reliées plus à des interrogations sociales qu'à des solutions techniques» (Hegedüs, 1985).

Mais un peu plus tard, les tensions sociales de 1968 et le choc pétrolier des années 70 catalysèrent toute l'attention. L'origine de ces bouleversements venait de loin. Le capitalisme industriel, nourri de machines et d'économies d'échelle, à la base de la course au progrès continu mais aussi aux contradictions croissantes entre le social et l'économie, voyait arriver ses propres limites.

En fait, on sait que chaque système a besoin de regénérer sa marge de manoeuvre, même au prix de sa nature propre. Un tel bouleversement avait déjà eu lieu au temps de la proto-industrialisation en faisant appel aux racines profondes du capitalisme.

De même la nouvelle mutation réclamait de nouvelles combinaisons entre le capital, le travail et l'énergie, en valorisant le capital au détriment des deux autres facteurs. Ce qui bousculait la division traditionnelle du travail et générait un processus capable de transformer graduellement l'ensemble du mode de production (Duché et Savey, 1985).

Il n'y a pas d'autres façons de conserver la «centralité» de la production que celle de faire évoluer ses agents économiques vers une production immatérielle, pour ainsi faire émerger une nouvelle qualité productive avec le minimum de dépense énergétique. En transférant ainsi le moteur de la production des biens aux services et en rendant ceux-ci prioritaires et autonomes, le leadership du processus productif passe à la recherche et aux services.

Ce processus, à peine créé, s'accélère. Rapidement, non seulement l'ensemble de la production, mais encore son soutien social, développent différents types de technologies qui interagissent entre elles. La nouvelle gamme de produits et de modes des productions qui s'en suit modifie et finit par transformer le style de vie. 
La technologie provenant du développement des connaissances scientifiques tend à se diffuser de plus en plus au niveau international sinon global (Colombo, 1985). En même temps, la production acquiert une nouvelle flexibilité pour répondre de façon plus appropriée aux conditions du marché, aux traditions, à la culture et autres éléments favorisant son adoption. Le nouvel environnement économique créé, en particulier grâce à l'informatique, facilite la recomposition de la fabrication selon ses différentes forces essentielles (Martinelli, 1986). La nouvelle technologie connaît un rythme d'évolution trépidant qui réduit le cycle de vie commerciale des produits, en accélère la diffusion, permet d'élargir les champs d'applications et produit des biens améliorés.

La révolution informatique a aussi un impact général sur toute l'activité économique, de la conception à la stratégie globale. Elle permet une nouvelle approche, de type systémique, entre les agents et la décision, entre la gestion et le contrôle du processus de production. Enfin, une des caractéristiques, qu'on doit regarder de plus près, est sa capacité à discriminer tant les entreprises que les régions économiques. L'entreprise plus faible réussit difficilement à passer d'une stratégie réactive - ou complètement passive - à une stratégie adaptée ou orientée vers la recherche et le développement ou vers l'extension des nouvelles technologies.

Cette période d'innovation devient une variable stratégique, encore plus centrale si elle est reliée à tout le système d'information. Plus le processus productif devient complexe, plus les exigences de produire, de gérer et d'évaluer le flux continu d'informations de sources diverses augmentent (Menegatti, 1984). Ce processus de multiplication de l'information à rechercher pousse la société à contrôler plus rigoureusement les canaux qui en diffusent et en filtrent le flux. Dans ces conditions, enfermer l'économie sur la base de la division traditionnelle entre le secondaire et le tertiaire devient un exercice à peu près impraticable. La fonction du tertiaire intermédiaire entre le processus productif et son environnement ne peut contribuer qu'à rendre plus flexible et plus souple le système industriel (Vaccà, 1980; Rullani, 1980).

Sous cet angle, les nouvelles typologies basées sur un continuum entre la production de biens et de services deviennent un instrument fondamental de l'analyse régionale. Une classification réalisée à cette fin par Bailly (1985) propose par exemple, que les activités servant de référence au «système de production» soient divisées selon les quatre fonctions fondamentales : fabrication, circulation, distribution et régulation. Une telle division «par fonction» permet de contourner la rigidité habituelle des statistiques, pour mieux voir les tendances actuelles du système de production; celles-ci permettent de préciser «la nouvelle importance de la circulation, la spécialisation spatiale des centres d'activités pour la distribution et pour la régulation des flux, et, en périphérie, la concentration de la fabrication/distribution». Ces éléments peuvent être traités dans une grille qu'on veut plus éloquente sur la potentialité de la fonction d'organisation des points centraux et la confirmation de la fonction d'exécution assignée aux espaces périfériques. 
Ces transformations qui découlent de la nouvelle géographie industrielle permettent finalement de mieux spécifier les localisations, d'harmoniser «l'architecture» et d'éviter les ruptures entre les lieux administratifs. Une entreprise chef de file comme I.B.M. peut choisir de s'implanter dans la campagne; il en est de même pour les usines comme celle de Corbeil, comme le laboratoire de recherche de La Gaude (Bakis, 1977) ou encore pour le grand complexe de Yorktown (De Barbieux, 1985). Une cafétéria commerciale peut prendre le virage campagnard. On peut même résoudre le dilemne entre le respect des espaces verts et le besoin d'aires de piquenique sans tout bouleverser l'environnement comme le ferait un mastodonte (Sanger, 1985). Les «prisons inhumaines» de Max Weber, qui ont marqué l'histoire industrielle sont en déclin. Outre ce questionnement en profondeur entre l'exploitation des ressources et le respect de l'environnement, le progrès technologique a entraîné de nouvelles obligations pour l'activité productive, a accéléré notamment le développement des transports et a permis de nouvelles formes de localisations. Il a multiplié le nombre de choix possibles (Malecky, 1983; Momigliano et Dosi, 1983).

La ré-occupation des espaces de production, dans un jeu accéléré de décomposition/recomposition des segments de production, transforme les équilibres oligopolistiques et concurrentiels (Martinelli, 1986). On n'assiste pas à un nouveau processus, mais à un bouleversement à travers lequel se manifestent des forces conflictuelles et l'orientation du capital permettant une nouvelle confrontation entre espaces industriels et régions encore peu touchées par le phénomène industriel.

Dans certaines régions, on assiste à l'arrivée de grandes entreprises multinationales qui élargissent la gamme des productions et entrainent la différenciation et une nouvelle articulation territoriale autrefois limitées aux espaces centraux. Dans d'autres, c'est la marée montante de petites et moyennes entreprises qui s'approprient des parts de production de façon surprenante, ou des créneaux technologiques permettant des nouvelles spécialisations ou même des productions autrefois réservées aux grandes entreprises. Ces activités d'externalisation ou de pénétration des «interstices» permettent de développer les espaces périfériques sur une base territoriale; cette dernière devient en quelque sorte autonome, développant les liaisons fonctionnelles internes qui lui confèrent la cohérence pour devenir un petit système.

Notre époque assiste donc au triomphe de la petite et moyenne entreprise, car elle est à la base, de façon incontestée, de la création des nouveaux emplois; elle attire ainsi l'attention des politiques industrielles gouvernementales. Ce phénomène est commun à toutes les puissances industrielles. Ainsi, aux Etats-Unis, depuis 1975, $80 \%$ de la croissance des emplois provient des entreprises de moins de 500 employés et la moitié de celles-ci ont moins de 20 employés (Colombo, 1985). En France, l'impôt sur l'emploi des dix dernières années est positif pour les entreprises de moins de 100 employés et négatif pour les autres (Duché et Savey, 1984). La même chose se passe en Grande-Bretagne. Au Japon, depuis longtemps, le tissu des petites et moyennes entreprises est étroitement relié aux grands groupes industriels ou aux colosses qui contrôlent le commerce extérieur; ceci comprend presque tous les secteurs et assure une base large et solide à la pyramide productive (Zanetto, 1986); ces 
dernières années, cette expansion des petites entreprises (de moins de 300 employés) a permis de compenser les pertes d'emplois provenant des grandes entreprises.

De plus en plus d'espaces périphériques où la main-d'oeuvre est disponible et où les déséconomies d'agglomération sont absentes attirent les nouveaux investissements industriels. Aux Etats-Unis, le processus de décentralisation territoriale vers des zones moins congestionnées s'est accentué depuis le second conflit mondial (Norton et Rees, 1978). Déjà, après la guerre, les zones industrielles traditionnelles du Nord-Est et du haut Midwest, qui concentraient à peu près les trois-quart du potentiel productif, commencent à décliner. Après 1969, le phénomène s'accélère et oriente vers les périphéries presque la moitié des nouveaux emplois et de la valeur ajoutée. On assiste au déplacement graduel des «industries nucléaires motrices», comme la production d'équipements industriels ou de produits électroniques. La capacité innovatrice émigre vers le Sud et vers l'Ouest, régions qui cessent d'être de simples régions exportatrices de matières premières, de viandes en boîte et de jus d'orange. Petit à petit, l'évolution de la haute technologie vers la périphérie se généralise; même Los Angeles, la zone centrale de l'Ouest, perd des emplois créés durant les années 70 au profit des zones moins centrales, comme San José, Anaheim et San Diego.

Dans d'autres pays occidentaux, le renversement des rapports centrepériphérie qui accompagne la relocalisation industrielle semble plus systématique. Ainsi, en Grande-Bretagne, les nouvelles implantations des multinationales dans l'électronique et dans de nombreux autres secteurs se font dans les zones industrielles périphériques jusqu'en Ecosse, transformant le centre innovateur des grandes zones industrielles urbaines; les petites et moyennes entreprises manufacturières de l'OuestMidlands survivent à la récession et se reconvertissent vers la robotique et l'électronique de pointe (Oakey et alii, 1980).

En France, les années 70 voient le glissement du centre industriel vers l'ouest de la diagonale Bretagne-Languedoc et l'accélération de la crise dans les vieux bassins industriels du Nord et de l'Est (un peu moins en Touraine). On assiste à une relocalisation vers des régions sans passé industriel qui affichent un haut taux de chômage, qui offrent des salaires et des qualifications modestes ainsi qu'une possibilité d'intégration des pratiques agricoles. De plus, la forme dominante des entreprises des nouvelles zones industrielles passe par l'éclatement en de multiples petites et moyennes entreprises localisées à proximité des centres secondaires. Toutefois, cette évolution n'a pas encore affecté de façon substantielle les centres moteurs en innovation de l'industrie française (Guglielmo, 1981).

Pour la nouvelle Italie émergeant des années 70, les économistes ont dû abandonner le schéma dualiste (Nord/sud) développé systématiquement au cours des deux décennies précédentes suite à la phase de concentration spatiale démarrée après-guerre. On découvre la Troisième Italie (Bagnasco, 1977), mais aussi plus que cela. On assiste à un nouveau dynamisme des petites et moyennes entreprises non toujours relié à la diversification des grandes entreprises en crise (Goglio, 1982). 
Aux modèles interprétatifs néo-classiques, fondés sur la fluidité absolue interrégionale du capital et du travail, on oppose des modèles plus raffinés des «filtering down» basés sur l'hypothèse d'une redistribution hiérarchique de l'activité manufacturière, soit du cycle de production à celui de processus de production, jusqu'à celui de processus d'innovation. En quelque sorte, on assiste au déclin d'un monde économique dans lequel «la pression concurrentielle écrasait tout jusqu'à sa frontière - encore mouvante - technologique» (Becattini et Bianchi, 1985 : 32). L'époque contemporaine voit l'apparition d'un marché de travail segmenté et d'un marché de biens de plus en plus complexe, la difficulté d'enfermer l'entreprise dans ses frontières, mais surtout le développement de forces d'inertie et de nouveaux espaces disponibles pour l'aménagement.

Mais pour comprendre ce nouvel environnement, il manque un élément fondamental qui doit permettre de spécifier le potentiel d'aménagement d'une zone et de comprendre les liens entre l'échelle d'analyse et celle à laquelle opèrent les forces économiques et sociales acquises dans une phase déterminée. C'est pourquoi on doit passer d'un niveau de lecture qui spécifie la séquence hiérarchique entre les zones de production forte et faible à un autre schème d'interprétation moins réducteur. En substance, on doit parvenir à une analyse plus sensible de la réalité géographique qui met de côté les données statistiques de localisation spatiale pour tenir compte de façon importante - sinon de façon centrale — du territoire et de son histoire. La région et le processus de régionalisation deviennent des protagonistes de cette nouvelle approche interprétative.

Peut-on comprendre les grands bouleversements de l'Italie sans aborder l'évolution de son développement à la lumière de réflexions économiques, géographiques et sociales ? C'est la seule façon de comprendre une spécifité léguée par une situation plus floue, moins «efficiente» par rapport à des économies plus solides du capitalisme avancé. Dans une Italie qui n'a pas encore absorbé tous les aspects du modernisme de la phase actuelle de la croissance industrielle, la fin des années 70 présente de nombreuses et fortes tensions internes, spécialement sur le marché du travail dans les zones riches et face au risque de la concurrence des nouveaux produits internationaux. La crise s'abat en premier lieu sur la grande entreprise, mais elle affecte tout le tissu social et limite la capacité de médiation de l'Etat. Dans le reste de l'Europe industrielle, la réponse qui doit passer par le réalignement territorial (en terme de grandes infrastructures, des services et de complexes de recherche) pour rencontrer les exigences de la partie la plus avancée du capital, se fait en retard ou de façon trop timide.

Les difficultés rencontrées par la grande industrie se reflètent sur tout le secteur secondaire. Entre les deux derniers recensements (1971 et 1981), l'emploi manufacturier s'élargit de façon continue alors qu'il stagne dans le complexe du triangle industriel. Pendant ce temps, les zones qui dépassent la croissance moyenne nationale se multiplient, poursuivant la tendance de longue période (fig. 1). La première conséquence de cette tendance est la perte de vitesse des zones manufac- 
Fig. 1 La dynamique de l'industrie manufacturière*

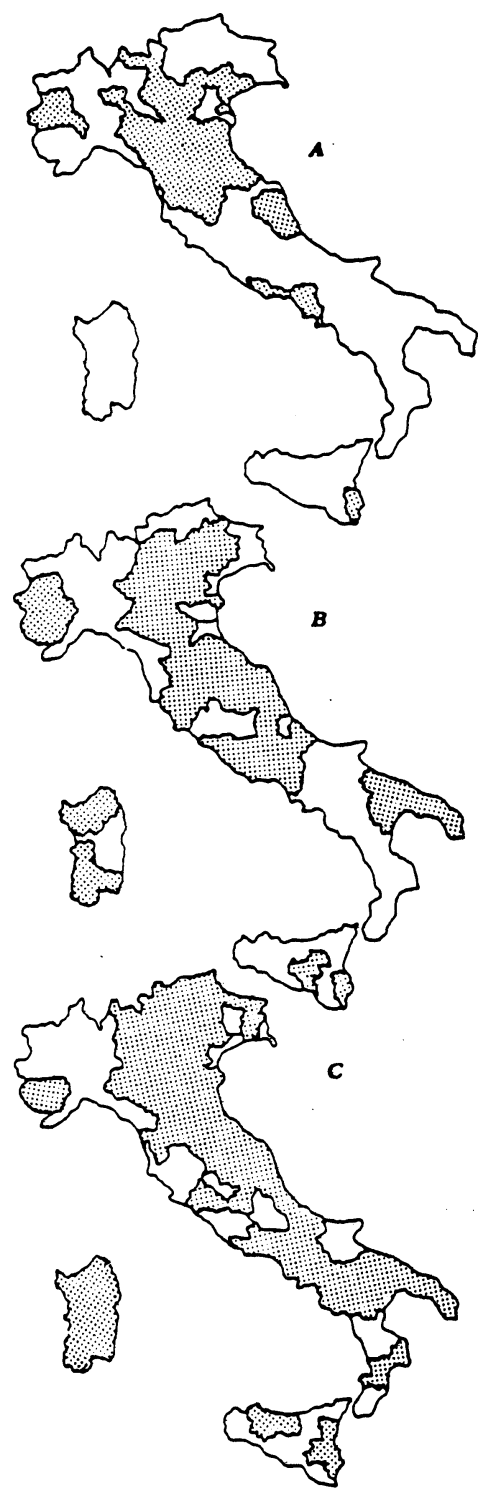

- Sur chaque carte figurent les provinces dont le taux de croissance des revenus est supérieur à la moyenne nationale pour les périodes de A) 1951-61, B) 1961-71 et C) 1971-81. 
turières historiques et du processus de concentration. Déjà présente à la fin des années 70 , cette tendance s'accentue fortement ces derniers temps.

Le déclin des régions du Nord-Ouest dérive de la chute verticale des niveaux d'emploi des grandes entreprises (plus de 1000 employés). Pour tout le pays, l'emploi dans celles-ci diminue en moyenne de $10 \%$ entre les derniers recensements; cette baisse se poursuit par la suite à un rythme estimé de $13 \%$ dans les trois années suivantes (Altieri, 1985). Ce retard de la grande industrie fait ressortir le dynamisme des petites entreprises manufacturières dans certaines mesures déjà localisées ou tendant à s'installer toujours plus à la périphérie, en réponse aux «conditions territoriales spécifiques». Durant les dix dernières années, de telles initiatives permettent de créer de l'emploi à un taux supérieur à celui généré par les grandes entreprises; cela est encore plus vrai pour la Toscane, le Veneto, et surtout pour la Marche. Ce développement de l'emploi est favorisé par une remarquable flexibilité en terme de force de travail, d'appareils technico-commerciaux et de dotations financières. Une large ouverture vers l'extérieur, avec une part de produits destinés à l'exportation presqu'aussi importante que celle destinée au marché intérieur, atteste d'une vitalité particulière et s'explique par des crénaux ou la recherche d'interstices sur les marchés.

L'émergence de «l'Italie du Centre» permet donc une certaine généralisation du «welfare state» et une certaine péréquation en infrastructures physiques et sociales (Dematteis, 1985; Fuà, 1983). On assiste aussi à une meilleure relation entre l'offre et la demande locale de travail, une consolidation des structures économiques et un renforcement des traditions culturelles et du patrimoine historique.

L'histoire récente de cette partie de l'Italie qui a forgé le marché NEC(NordEst-Centre) est faite surtout d'actions entreprises par les agents publics et privés pour contrôler l'innovation territoriale et pour introduire les technologies et les formes d'organisation pouvant facilement se conjuguer avec le milieu local. Elle repose sur un processus diffus mais fragmenté, avec plusieurs protagonistes peu ou non coordonnés, visant à saisir les possibilités accumulées durant de longues périodes (par exemple, la forme de tenure des terres et de l'organisation rurale); elle a permis de profiter des nouvelles opportunités ouvertes par le redéploiement des grandes métropoles (ou mieux leur épuisement en faveur d'autres objectifs et niveaux territoriaux). Ce processus s'explique par le besoin de modernisation face à des éléments et séquences propres à la proto-industrialisation, telles les règles (sociales et non seulement économiques) familiales, la fréquence du travail à domicile et la dispersion de l'industrie dans la campagne. Les caractéristiques de ce processus touchent aussi au système de valeur, profitant de plusieurs itinéraires secondaires ou alternatifs que l'histoire et la théorie du développement tournées toujours vers la grande dimension, semblaient avoir relégué à la marge.

Il n'est pas question ici, cependant, de revenir sur les analyses des conditions favorables qui ont permis de soutenir et de faire décoller l'Italie périphérique. Ces questions sur les structures territoriales et les facteurs favorisant le décollage sont très 
intéressantes. Mais il est préférable de présenter le cadre des changements au moins aussi important dans la combinaison des facteurs, des secteurs de productions et des formations sociales, afin d'expliquer ce qu'on a appelé, sous une vague étiquette, «l'Italie du Centre».

Entre les périphéries émergentes, le Veneto occupe une place spéciale. C'est là qu'on a connu la plus forte croissance des revenus et l'extension la plus soutenue de la production locale. C'est encore la région où on est le plus proche du modèle de l'industrialisation diffuse (Lando et Zanetto, 1986). Elle constitue peut-être le meilleur exemple pour comprendre le concept d'ensemble et développer un minimum d'éléments paradigmatiques. La force de l'industrie du Veneto, pour l'exportation, dans le meuble et la petite mécanique, démontre une vitalité particulière (comme mode de système), celle-ci surpasse en valeur la production moyenne nationale. Cette capacité commerciale pour affronter la concurrence se fonde sur un très bon mécanisme de mise à jour des produits et de la distribution. C'est ainsi que les petites et moyennes entreprises innovatrices réussissent à développer une bonne stratégie, à produire des biens finis de très bonne teneur, à travailler sur de l'assemblage ou sur des petites séries et à miser sur la qualité, tout en réussissant, toujours en périphérie, à concilier la recherche et les besoins du marché. La relative indifférence d'une telle production pour la localisation permet de favoriser dans ces circonstances «un environnement qui par la qualité de vie, la tranquilité syndicale et l'éthique de travail, facilite le support exteme des travailleurs et des familles et le soutien dans les périodes difficiles du démarrage ou lorsque l'entrepreneur innove en courant des risques majeurs» (Rizzo et Robiglio Rizzo, 1988 : 433). L'ensemble des entreprises profite des structures diffuses offrant une quantité satisfaisante de services tertiaires, sans polarisation excessive ou non limités par les cinq centres majeurs de la région. Ce modèle polycentrique est en quelque sorte encouragé encore par une gestion politique dont l'objectif est d'atténuer les conflits de classes et d'éviter ainsi toutes tendances à une segmentation sociale.

Le manque de forte polarisation ne veut pas dire du tout le développement de déséquilibres, comme par exemple entre la partie centrale plus dynamique et les zones externes laissées au tourisme et à l'agriculture. L'élément le plus intéressant, pour les conséquences qui peuvent surgir à moyen terme de cet aménagement territorial, est d'abord un contraste de niveau : à un vieux réseau de relations provinciales en voie de démantèlement se superpose, en effet, un nouveau modèle d'exigences et de relations axées sur des activités plus adéquates et plus innovatrices. Ces deux niveaux qui s'interpénètrent permettent probablement d'éviter toute faillite du territoire et de favoriser la maturation du modèle vénitien (Zanetto, 1988).

Un des éléments sur lesquels se mesure le potentiel d'identification et les forces organisatrices de chaque zone périphérique est cette force qui permet au potentiel «collectif» favorisant la promotion de l'innovation de s'exprimer dans les activités caractéristiques. Ceci n'est qu'un exemple - mais assez significatif — du climat social général qui facilite les comportements «publics» internes à la base de 
l'économie locale. Il repose en grande partie sur les traditions municipales et la forme articulée de participation sociale qui est spécifique à la périphérie italienne.

Nous pouvons trouver d'autres exemples dans deux zones textiles désormais consolidées, soit Prato en Toscane et Biella dans le Piémont. Tant par le contexte géographique que par le type et la taille des firmes productives, ces deux districts industriels ont été des précurseurs dans la phase de reconversion de l'ancienne industrie textile à partir d'une fragmentation poussée du cycle productif et une série d'innovations dans les techniques et la nature des produits (par exemple, la nouvelle carde de Prato) ou dans de nouveaux débouchés (par exemple, l'option de moyenne gamme et de vêtement sportif à Biella).

Cette évolution s'explique par le rôle central des petits entrepreneurs disposés à affronter les risques du futur. Mais un autre point spécifique complexe qui distingue ces zones et qui repose sur leur capacité de profiter de nouveaux «acteurs horizontaux» vient des liens créés entre les institutions financières et les associations d'entreprises dans le but de développer de nouvelles compétences technologiques et managériales. Pour le Prato, cela passe par les services de recherche de la «technotextile» et les relations avec le système videotex (le projet SPRINT). Biella, pour sa part, a mis sur pied le grand projet de la «Cité des études sur le textile» qui intègre les aspects de recherche et de formation professionnelle.

Ces stratégies peuvent permettre de renforcer certains systèmes productifs locaux pour plus d'ouverture et plus d'innovation; elles facilitent «l'usage rapproché et savant d'un complexe d'économies externes, d'échelle et d'agglomération, pour les intégrer à une structure productive fonctionnellement déconcentrée et contemporainement accentuée dans une zone-système fortement intégrée» (Segre, 1989).

Le développement d'expériences accumulées dans ces deux solides bassins textiles a permis la stimulation de l'intégration de plusieurs zones monoproductives en difficulté en profitant de ces nouvelles forces ou moyens. Elle a facilité la consolidation de l'appareil productif et a permis de faire un pas en avant pour renforcer les services à la production et éviter l'affaiblissement du système de valeur et du niveau d'autonomie. Une des limites qui risque de ronger les marges de reproduction du système peut provenir des changements sociaux et donc compromettre les précieux équilibres internes socio-territoriaux forgés tout au long de l'histoire (Tinacci, 1984).

Nul besoin de rappeler que cette spécificité socio-territoriale est le résultat d'un équilibre délicat, au coeur même du système, qui exprime un potentiel d'identité et d'autonomie et qui facilite la relève politique, laquelle est à la base des conditions de fonctionnement de l'administration locale et des tractations avec le pouvoir central (Tarrow, 1977; Trigilia, 1986).

Le niveau d'indépendance relative dont jouissent chacune des zones décentralisées, et encore plus chacune des zones-systèmes à caractère monoproductif, est aussi en rapport étroit avec les conditions territoriales qui distinguent l'Italie 
émergente des années 70 (Garofoli, 1981). Ses périphéries, en somme, seraient plus libres, plus créatrices et plus localisées que celles des autres régions de l'Europe occidentale; ou - si on préfère - moins dépendantes, moins répétitives, moins spatialement anonymes.

\section{BIBLIOGRAPHIE}

Altieri, G., (1985), «Evoluzione dell'occupazione industriale nelle grandi imprese»» Congrès national CGIL sur les grands groupes industriels, Modena, sept.

Bagnasco, A., (1977), Tre Italie. La problematica territoriale dello sviluppo italiano, Bologna, Il Mulino.

Bailly, A. S., (1985), «Le rôle des activités de service dans le développement régional suisse», Ann. Géogr., 94, pp. 257-269.

Bakis, H., (1977), IBM. Une multinationale régionale, Grenoble, Presses Univ. de Grenoble.

Becattini, G. et Bianchi, G., (1985), «Analisi dello sviluppo multiregionale versus analisi multiregionale dello sviluppo» dans G. Bianchi et Magnani (éds), Sviluppo multiregionale : teorie, metodi, problemi, Milano, Angeli, pp. 29-38.

Colombo, U., (1985), «Il nostro futuro energetico», Prometeo, 3, n 11, pp. 74-9.

De Barbieux, B., (1985), «I.B.M. à la campagne : l'évolution du Dutchers County (Etat de New York), 1940-1984», Ann Géogr., 94, pp. 270-97.

Dematteis, G., (1985), «Contro-urbanizzazione e deconcentrazione : un salto di scala nell'organizzazione territoriale», dans R. Innocenti (ed), Piccola città e piccola impresa, Milano, Angeli, pp. 101-18.

Duché, G. et Savey, S., (1985), «Nouvelle organisation de la production et PME. Vers un nouveau système industriel», Bulletin de la Société Languedocienne de Géographie, 1-2, pp. $21-42$.

Fuà, G., (1983), «L'industrializzazione nel Nord-Est e nel Centro», dans G. Fuà et C. Zacchia (éd.), Industrializzazione senza fratture, Bologna, Il Mulino, pp. 7-46.

Galbraith, J.K., (1958), The affluent Society, Boston, Houghton Mifflin Co.

Garofoli, G., (1981), «Lo sviluppo delle «aree periferiche» nell'economia italiana degli anni Settanta», L'Industria, 3, pp. 391-404.

Goglio, S., (1982), Italia : centri e periferie, Angeli, Milano.

Guglielmo, R., (1981), «Le redéploiement industriel en France», Hérodote, n² 23, pp. 33-69.

Hegedüs, Z., (1985), Il presente è l'avvenire. Nuove pratiche e nuova rappresentazione sociale, Milano, Angeli.

Lando, F. et Zanetto, G., (1986), «L'industria veneta : dinamica della distribuzione geografica", dans C.U.D.A., Organizzazione del territorio ed evoluzione del sistema produttivo veneto, Altavilla Vicentina, pp. 214-366.

Malecky, E.J., (1983), «Science, Technology and Regional Development : a Survey», Intern. Reg. Sc. Rev.8, pp. 89-125.

Martinelli, F., (1986), «Tecnologia e sviluppo», La nuova città, 1, n¹, p. 14-30.

Menegatti, B., (1984), «Aspetti della teoria dell'informazione e della geografia della communicazione con particolare riferimento al terziario avanzato. Argomenti per la discussione», dans AA. VV., Strutture Industriali - Reti informative avanzate, Seminaire sur l'information, pp. 44-56.

Momigliano, F. et Dosi, G., (1983), Techologia e organizzazione industriale internazionale, Bologna, Il Mulino. 
Norton, R.D. et Rees, J., (1979), «The Product Cycle and the Spatial Decentralization of American Manufacturing», Reg. Studies, 13, pp. 141-51.

Oakey, R.P., Thwaites, A.T., et Nash, P.A., (1980), «The regional distribution of innovative manufacturing establishments in Britain», Reg. Studies, 14, pp. 235-53.

Rizzo, G. et Robiglio Rizzo, C., (1986), La piccola industria innovativa nelle aree a industrializzazione diffusa, dans P.Coppola (éd.), Atti XXIV Congr. Geogr. Ital., Torino, vol. 2.

Rullani, E., (1980) «Crisi della produttività industriale e sviluppo del terziario», Econ. e pol. industr., $\mathrm{n}^{\circ} 25$, pp. 209-22.

Sanger, D.E., (1985), «Un cliente piccolo ha trasformato il gigante», Genius, n 13, pp. 58-66.

Segre, A., (1988), «Innovazione, nuovo ambiente d'impresa e territorio in alcune aree tessili italiane», dans P. Coppola, (éd.), Soggeti economici e gerarchie territoriali, Patron, Bologna.

Tarrow, S., (1977), Between Center and Periphery, New Haven, Yale Univ. Press.

Trigilia, C., (1986), Grandi partiti e piccole imprese, Bologna, Il Mulino.

Vaccà, S., (1980), «Un nuovo terziario per trasformare l'industrializzazione», Econ. e pol. industr., 6, $\mathrm{n}^{\circ} 25, \mathrm{pp} .11-28$.

Zanetto, G., (1986), «Innovazione tecnologia e localizzazione nei paesi OCDE e, in particolare, in Giappone», dans AA. VV., Venezia città dell' innovazione, Venezia, Marsilio, pp. 111-46. 